\title{
Giardiasis en Perros Menores de Tres Años que Concurren a los Parques Públicos del Distrito de Santiago de Surco en Lima Metropolitana
}

\author{
Giardiasis in Dogs under Three Years of Age that Go to Public Parks in the \\ District of Santiago de Surco in Lima
}

Fiorela Huamancayo L. ${ }^{1}$, Amanda Chávez V. ${ }^{1,2}$

\section{RESUMIEN}

\begin{abstract}
El objetivo del estudio fue estimar la frecuencia de infección con Giardia sp en caninos menores de tres años que acuden a los parques públicos del distrito de Santiago de Surco de Lima Metropolitana y determinar la concordancia entre tres técnicas de diagnóstico. Se recolectaron muestras fecales de 140 caninos aparentemente sanos, menores de tres años de edad, que acudían a los parques públicos acompañados de sus propietarios. Para el diagnóstico de las muestras, se utilizaron las técnicas de inmunoensayo de cromatografía (IEC), la técnica de Faust (TF) y la técnica de sedimentación espontánea (TSE). Se encontraron frecuencias de infección con Giardia sp de 25.0, 17.9 y $12.1 \%$ con estas técnicas, respectivamente. El análisis de asociación entre la frecuencia de infección y las variables predictoras (sexo, grupo etario, estado de las heces o frecuencia de visitas por día a los parques) en un modelo de regresión logística no demostraron significancia estadística. Los índices de concordancia de Kappa entre las pruebas diagnósticas fueron 0.78 (IEC vs TF), 0.58 (IEC vs TSE) y 0.77 (TF vs TSE) ( $\mathrm{p}<0.05$ ). Los resultados indican un moderado nivel de infección con Giardia sp evidenciando un potencial riesgo zoonótico para la población humana. Adicionalmente, tanto la IEC como TF demostraron un nivel de concordancia substancial.
\end{abstract}

Palabras clave: Giardia sp, caninos, heces, parques públicos, Santiago de Surco, Lima

\footnotetext{
${ }^{1}$ Laboratorio de Microbiología y Parasitología Veterinaria, Facultad de Medicina Veterinaria, Universidad Nacional Mayor de San Marcos, Lima

${ }^{2}$ E-mail: achavezvg@gmail.com
}

Recibido: 10 de mayo de 2014

Aceptado para publicación: 16 de febrero de 2014 
The aim of this study was to estimate the frequency of infection by Giardia sp in dogs under three years of age that go to public parks in the district of Santiago de Surco, Lima, Peru, and to determine the concordance among three diagnostic techniques. Stool samples were collected from 140 apparently healthy dogs under 3 years old that went to public parks accompanied by their owners. For the diagnosis of the samples, three techniques were used: Chromatography Immune-essay (CIE), Faust's technique (FT) and Spontaneous Sedimentation Technique (SST). The results showed that 25.0, 17.9 and $12.1 \%$ of dogs were infected with Giardia sp according to the three techniques respectively. Analysis of association between the frequency of infection and the predictor variables (sex, age, physical status of the stools, number of daily visits to the park) in a logistic regression model did not show statistical significance. The Kappa indexes of concordance among the three diagnostic tests were 0.78 (CIE vs FT), 0.58 (CIE vs SST) and 0.77 (FT vs SST) $(\mathrm{p}<0.05)$. The results showed a moderate level of infection with Giardia sp indicating a potential of zoonotic risk to human population. In addition, both IEC and TF techniques showed a substantial levels of of concordance.

Key words: Giardia sp, canines, stool samples, public parks, Santiago de Surco, Lima

\section{INTRODUCCIÓN}

La giardiasis, parasitosis causada por el protozoo flagelado Giardia sp es el protozoario entérico más frecuente en el humano, estimándose que 280 millones de personas se encuentran afectadas (Thompson, 2008). La transmisión puede ser directa, a través de la vía fecal-oral, o indirecta, a través de la ingestión de agua o alimentos contaminados (Sprong et al., 2009). Tanto la infección como la enfermedad son más frecuentes en niños y animales jóvenes (Acha y Szyfres, 2003).

El parásito se ubica en el intestino delgado de perros, gatos, vacunos y otros mamíferos domésticos y silvestres (Thompson, 2008). Los signos clínicos se caracterizan por la producción de cuadros gastrointestinales que varían desde infección asintomática a enfermedad aguda o crónica asociado con diarrea y síndrome de mala absorción (Cordero del Campillo et al., 1999).

Se dispone de escasos estudios en el Perú sobre giardiasis canina, los cuales han sido llevados a cabo en la ciudad de Lima
Metropolitana y en la Provincia Constitucional del Callao, donde se reportaron prevalencias de 9.35 y $16.7 \%$ (Zárate et al., 2003; Araujo et al., 2004; Sotelo et al., 2013). Sin embargo, no existen estudios que contemplen la relación de caninos que acuden a las áreas de recreación como parques públicos y la presencia de Giardia sp en ellos, como un potencial riesgo zoonótico.

Por otro lado, el diagnóstico de giardiasis es complicado, debido la intermitencia en la eliminación de quistes del parásito (Carlin et $a l ., 2006)$ y la necesidad de personal experimentado en la detección de las formas parasitarias (OMS, 2002; Carlín et al., 2006), de allí que se han tenido que desarrollar diversos métodos inmunológicos y moleculares con mejores resultados (Fuentes et al., 2010).

Datos no publicados de la casuística del laboratorio de parasitología de la Facultad de Medicina Veterinaria (FMV) de la Universidad Nacional Mayor de San Marcos (UNMSM), Lima, han mostrado que mascotas caninas provenientes de distritos socioeconómicos altos presentan elevadas frecuencias de giardiasis al examen 
laboratorial. Además, se conoce que el sistema inmunitario en animales jóvenes está en proceso de maduración, de allí que estos son susceptibles a adquirir diversas enfermedades. Por esto, el objetivo del presente estudio fue estimar la frecuencia de Giardia sp en caninos jóvenes que concurren a los parques públicos de un distrito de clase media alta del sur de Lima Metropolitana, así como determinar la concordancia entre tres técnicas de diagnóstico.

\section{Materiales y Métodos}

El estudio se realizó en el distrito de Santiago de Surco, de la ciudad de Lima, Perú. La zona presenta un clima templado con temperaturas máximas de $27^{\circ} \mathrm{C}$ y mínimas de $15{ }^{\circ} \mathrm{C}$, y con una humedad relativa cercana al 95\% (INEI, 2007). El muestreo se realizó en los parques públicos del distrito entre setiembre de 2011 y enero de 2012.

El tamaño muestral $(n=130)$ se determinó mediante la fórmula para estimar una proporción para poblaciones infinitas (Daniel, 1996), utilizando una prevalencia referencial de $9.35 \%$, hallada por Araujo et al. (2004). No obstante, se logró trabajar con 140 canes.

Se seleccionaron perros aparentemente sanos, entre un mes y tres años de edad, sin restricción de sexo y raza, residentes de la zona, que hayan acudido con sus propietarios a los parques públicos y hayan brindado las facilidades para la toma de muestra de heces.

Se trabajó en 50 parques públicos. Se recolectaron cerca de $30 \mathrm{~g}$ de heces recién evacuadas y de la porción superficial, libre de contacto con el suelo. Se identificó el nombre, sexo, edad y raza del animal, y frecuencia de salida al parque. Los frascos fueron almacenados en cajas conservadoras con refrigerante, y transportadas al Laboratorio de Parasitología de la FMV-UNMSM.
Para la evaluación coproparasitológica se empleó la técnica de inmunoensayo de cromatografía (IEC), usando el kit comercial para detección de antígeno de Giardia (Anigen Rapid Giardia Ag Tes Kit, BioNote ${ }^{\circledR}$ ), la técnica de Faust (TF) o técnica de flotación con sulfato de zinc al 33.3\% (Beltrán et $a l ., 2003)$ y la técnica de sedimentación espontánea (TSE) (Tello, 1988).

La frecuencia de giardiasis se expresó en forma porcentual, con sus respectivos intervalos de confianza al $95 \%$. Se evaluaron las variables sexo, estado físico de las heces (formadas, pastosas), edad (menor de 6 meses, de 6 meses a 1.5 años, mayor de 1.5 a 3 años) y frecuencia de visitas al parque (1, 2 y más de dos veces por día).

Se utilizó la prueba de regresión logística para evaluar la fuerza de asociación entre las variables de interés con un nivel de significancia de 0.05 , así como la prueba de Kappa para evaluar la concordancia entre las técnicas de diagnóstico. Los datos fueron procesados mediante el paquete estadístico SPSS v. 18.6 para Windows.

\section{Resultados}

La frecuencia de Giardia sp fue de 25.0 $\pm 7.2 \%$ mediante la técnica de IEC (Cuadro 1). La frecuencia de animales positivos fue mayor en machos $(30.8 \%)$ que en hembras $(14.3 \%) \quad(p<0.06)$. Asimismo, hubo ligeras diferencias entre edades, estado físico de las heces y frecuencia de visitas al parque, pero sin diferencia estadística.

En el Cuadro 2 se muestra la frecuencia de Giardia sp en caninos según las técnicas de diagnóstico empleadas. El mayor número de casos positivos fue detectado con la IEC (25\%) y la menor cantidad de animales positivos fue con la técnica de sedimentación espontánea (12.1\%). Los índices de concordancia de Kappa entre las pruebas 
Cuadro 1. Frecuencia de Giardia sp en caninos menores de tres años que concurren a los parques públicos del distrito de Santiago de Surco, Lima, determinado por la técnica de inmunoensayo de cromatografía (2012)

\begin{tabular}{|c|c|c|c|c|c|}
\hline \multirow[b]{2}{*}{ Variable } & \multicolumn{2}{|c|}{ Muestreados } & \multicolumn{2}{|c|}{ Odds Ratio (OR) } & \multirow[b]{2}{*}{$\mathrm{p}$} \\
\hline & N. ${ }^{\circ}$ & $\begin{array}{c}\text { Positivos } \\
(\%)\end{array}$ & OR & $\begin{array}{c}\mathrm{IC}^{1} \text { del } \\
95 \% \\
\end{array}$ & \\
\hline \multicolumn{6}{|l|}{ Sexo } \\
\hline Hembra & 49 & 14.3 & 1 & & \\
\hline Macho & 91 & 30.8 & 2.5 & $0.9-6.6$ & 0.06 \\
\hline \multicolumn{6}{|l|}{ Edad (años) } \\
\hline$<0.5$ & 20 & 25.0 & 1 & & \\
\hline $0.5-1.5$ & 49 & 30.6 & 0.8 & $0.2-2.7$ & 0.74 \\
\hline$>1.5-3$ & 71 & 21.1 & 1.4 & $0.4-4.9$ & 0.55 \\
\hline \multicolumn{6}{|c|}{ Estado físico de las heces } \\
\hline Formadas & 70 & 20.0 & 1 & & \\
\hline Pastosas & 70 & 30.0 & 1.7 & $0.7-3.9$ & 0.20 \\
\hline \multicolumn{6}{|c|}{ Visitas al parque por día } \\
\hline 1 & 38 & 21.2 & 1.6 & $0.5-4.8$ & 0.33 \\
\hline 2 & 47 & 21.3 & 1.6 & $0.6-4.1$ & 0.30 \\
\hline$>2$ & 55 & 30.9 & 1 & - & \\
\hline Total & 140 & $25.0 \pm 7.2$ & & & \\
\hline
\end{tabular}

${ }^{1}$ Intervalo de confianza

Cuadro 2. Frecuencia de Giardia sp en 140 caninos menores de tres años del distrito de Santiago de Surco, Lima, según técnica de diagnóstico (2012)

\begin{tabular}{lcc}
\hline \multirow{2}{*}{$\begin{array}{l}\text { Técnica de } \\
\text { diagnóstico }\end{array}$} & \multicolumn{2}{c}{ Positivos } \\
\cline { 2 - 3 }$\% \pm \mathrm{IC}^{1}$ \\
\hline $\begin{array}{l}\text { Inmunoensayo de } \\
\text { cromatografía }\end{array}$ & 35 & $25.0 \pm 7.2$ \\
$\begin{array}{l}\text { Técnica de Faust } \\
\text { Sedimentación }\end{array}$ & 25 & $17.9 \pm 6.3$ \\
espontánea & 17 & $12.1 \pm 5.4$ \\
\hline${ }^{1}$ Intervalo de confianza & &
\end{tabular}

diagnósticas fueron de 0.78 para IEC vs TF, 0.58 para IEC vs TSE y 0.77 para TF vs TSE, habiendo significancia estadística en todos los casos $(\mathrm{p}<0.05)$.

\section{Discusión}

La giardiasis es un importante problema de salud pública en el país debido a la alta prevalencia en humanos, especialmente en niños (Chincha et al., 2009; Alvarado et al., 2011). El estudio pretendió demostrar la presencia de Giardia sp en caninos que acuden 
a áreas de recreación como parques públicos.

Los resultados del estudio demostraron un $25 \%$ de frecuencia de Giardia sp en caninos que concurren a los parques públicos al utilizar la técnica de IEC. No obstante, los resultados en base a la prueba de sedimentación espontánea (12.1\%) son similares a estudios previos en varios distritos de Lima Metropolitana y en la Provincia Constitucional del Callao, que reportaron prevalencias de 9.35 a $16.7 \%$ utilizando dicha técnica (Zárate et al., 2003; Araujo et al., 2004; Sotelo et al., 2013). La técnica de sedimentación espontánea tiene una sensibilidad de $70 \%$, en tanto que sensibilidad de la técnica de IEC es del 98\% (Fuentes et al., 2010). Esto permitió poder detectar un mayor número de casos positivos con la técnica de IEC, que podrían haber pasado desapercibidos con el uso de otras técnicas.

Por otro lado, las características de la población canina en estudio, como son los caninos menores de tres años, permitían presagiar una mayor frecuencia de casos positivos por ser cachorros más propensos ser infectados con este protozoo (Epe et al., 2010). Es posible que el cuidado de los dueños, tratamientos de desparasitación y factores de saneamiento ambiental hayan permitido un mayor control de la giardiasis en estos canes.

No se encontró diferencia significativa en el presente estudio en la infección por Giardia sp por efecto del sexo, tal y como lo demuestran la mayor parte de la literatura científica (Araujo et al., 2004; Labarthe et al., 2008; Upjohn et al., 2010; Sotelo et al., 2013). Asimismo, la edad tampoco fue un factor determinante para la presencia del protozoo; resultados que concuerdan con Labarthe et al. (2008) en Rio de Janeiro, Brasil, quienes trabajaron con canes de hasta 2 años de edad; sin embargo, diversos estudios han demostrado que los individuos menores de 6 meses han sido los más afectados, debido posiblemente a la inmadurez de su sistema inmunológico (Cordero del Campillo et al., 1999; Epe et al., 2010; Sotelo et al., 2013).

El estado físico de las heces no fue un factor que afectó la identificación de casos de giardiasis. Upjohn et al. (2010) tampoco hallaron asociación entre la consistencia fecal y la infección por Giardia sp, indicando que habría igualdad de oportunidades de hallar el parásito en heces pastosas como en heces formadas. No obstante, existen estudios que señalan una mayor frecuencia de encontrar Giardia sp en heces pastosas, blandas y diarreicas (Zárate et al., 2003; Sotelo et al., 2013). Los resultados del presente estudio tienen particular importancia, ya que en la práctica clínica suele considerarse que las diarreas o heces blandas son compatibles con algún tipo de infección, incluyendo la infección por Giardia sp.

La frecuencia de presencia de los perros en los parques no estuvo asociado a la presencia de Giardia sp en las heces, aspecto que concuerda con el trabajo de Wang et al. (2012). Es conocido que el agua es el principal vehículo para la difusión de quistes de Giardia sp (Castellón et al., 1992), y los parques en evaluación son mayormente regados con aguas no potabilizadas, lo que podría constituir un medio de posible contaminación para los perros, especialmente para los que pasan más tiempo en ellos. No obstante, no se puede considerar el agua de regadío de estos parques como una fuente de contaminación hasta no hacer un análisis del agua o del suelo.

La técnica de IEC permite detectar una glicoproteína (antígeno específico de $65 \mathrm{kDa}$ ) presente en la pared de los quistes y trofozoítos de Giardia (Faubert, 2000). Debido a ello presenta un sensibilidad y especificidad de $100 \%$, tomando como «gold» estándar a la prueba de ELISA «SNAPPIDEXX» (Lieb y Zajac, 1999). Este factor permitió identificar más casos positivos que en las otras dos pruebas. Por otro lado, la intermitencia en la eliminación de quistes de 
G. lamblia (Carlin et al., 2006) es una de las causas probables de menor positividad observada con las técnicas de TF y TSE, pues solo se evaluó una muestra fecal por animal. Es posible que con un muestreo seriado se hubiera incrementado la sensibilidad de estas dos pruebas diagnósticas.

Mediante la prueba de Kappa se mostró una substancial concordancia $(k=0.78)$ al comparar IEC con TF. El índice de Kappa refiere correspondencia o conformidad entre los resultados de una prueba al compararla con la otra, pero no indica cual de las pruebas es más eficaz. La concordancia entre estas dos pruebas estaría favoreciendo a la TF ya que es una prueba económica y es el método de mayor elección para la detección de quistes de Giardia sp (Papini y Cardini, 2006). La sensibilidad de la TF varía de 50 a $70 \%$ cuando se realiza con una muestra, pero llega hasta $95 \%$ cuando se realizan muestreos seriados (Bartmann y Pacheco, 2004), sensibilidad muy cercana a la técnica de IEC.

\section{ConClusiones}

- La frecuencia de Giardia sp en caninos menores de tres años que concurren a los parques públicos del distrito de Santiago de Surco de Lima Metropolitana fue de $25.0 \pm 7.2 \%$, mediante la técnica de inmunoensayo cromatográfico.

- Las variables sexo, edad, estado físico de heces y frecuencia de visitas a los parques no mostraron asociación significativa con la presentación de Giardia sp.

- Se encontró una substancial concordancia $(k=0.78)$ con una muestra por animal entre los resultados de la técnica de inmunoensayo cromatográfico y la técnica de sedimentación espontánea.

\section{Agradecimientos}

Los autores agradecen la colaboración financiera del Consejo Superior de Investigaciones de la UNMSM para el desarrollo de esta investigación. Asimismo, al M.V.
Ernesto Mendiola por las facilidades brindadas por la empresa Pfizer para la realización del presente estudio.

\section{Literatura Citada}

1. Acha PN, Szyfres B. 2003. Zoonosis y enfermedades transmisibles comunes al hombre y a los animales. $3^{\text {a }}$ ed. Washington: OPS. 398 p.

2. Alvarado C, Paredes E, Contreras A, Ponte S. 2011. Estudio coproepidemiológico sobre parasitosis intestinal y su efecto en el estado nutricional antropométrico de los niños de Potao-Barranca. Portales médicos VI(14): 394 [Internet]. Disponible en: http:// www.portalesmedicos.com/revista/ vol06 n14.htm

3. Araujo W, Chávez A, Casas E, Falcón N. 2004. Prevalencia de Giardia sp en Canis faniliaris de la Provincia Constitucional del Callao. Rev Inv Vet Per 15: 145-150.

4. Bartmann A, Pacheco FA. 2004. Freqüência de Giardia lamblia em cães atendidos em clínicas veterinárias de Porto Alegre, RS, Brasil. Cienc Rural 34: 1093-1096.

5. Beltrán M, Tello R, Náquira C. 2003. Manual de procedimientos de laboratorio para el diagnóstico de los parásitos del hombre. Serie de normas técnicas N. ${ }^{\circ} 37$. Lima: Instituto Nacional de Salud. 90 p.

6. Carlin EP, Bowman DD, Scarlett JM, Garret J, Lorentzen L. 2006. Prevalence of Giardia in symptomatic dogs and cats throughout the United States as determined by the IDEXX SNAP Giardia test. Vet Ther 7: 199-206.

7. Castellón A., Reyes L, Chinchila M, Mora. D. 1992. Viabilidad de los quistes de Lamblia intestinales bajo diferentes condiciones. Rev Costarric Cienc Méd 13(1-2): 9-15.

8. Chincha O, Bernabé A, Samalvides $F$, Soto L, Gotuzzo E, Terashima A. 2009. Infecciones parasitarias intestinales y factores asociados a la infección por 
coccidias en pacientes adultos de un hospital público de Lima, Perú. Rev Chil Infectol 26: 440-444. doi: 10.4067/S071610182009000600008

9. Cordero del Campillo M, RojoVásquez FA, Martínez AR, Sánchez MC, Hernández S, Navarrete I, et al. 1999. Parasitología veterinaria. Madrid: McGraw Hill Interamericana. 968 p.

10. Daniel D. 1996. Bioestadística: base para el análisis de las ciencias de la salud. 5ª ed. México: Limusa. 878 p.

11. Effio TJ. 1998. Estudio preliminar del mercado veterinario peruano. N. ${ }^{\circ}$ Partida 0723-1998. INDECOPI. Lima, Perú.

12. Epe C, Rehkter G, Schnieder T, Lorentzen L, Kreienbrock L. 2010. Giardia in symptomatic dogs and cats in Europe. Results of a European study. Vet Parasitol 173: 32-38. doi: 10.1016/ j.vetpar.2010.06.015

13. Faubert G. 2000. Immune response to Giardia duodenalis. Clin Microbiol Rev 3:35-54.

14. Fuentes I, Gutiérrez M, Gárate T. 2010. Diagnóstico de las parasitosis intestinales mediante detección de coproantígenos. Enferm Infecc Microbiol Clín 28(Supl 1): 33-39.

15. [INEI] Instituto Nacional de Estadística e Informática. 2007. Censo de población y vivienda 2007. Población total, por sexo y lugar de empadronamiento, según distrito de Lima metropolitana. Lima, Perú. [Internet]. Disponible en: http://www.inei.gob.pe

16. Labarthe $N$, Mendes de Almeida $F$, Balbi M, Salomão M, Paiva J, Crissiuma A, et al. 2008. Prevalence of Giardia in household dogs and cats in the State of Rio de Janeiro using the IDEXX SNAP ${ }^{\circledR}$ Giardia test. Intern J Appl Res Vet Med 6: 200-206.

17. [OMS] Organización Mundial de la Salud. 2002: Protozoan parasites (Cryptosporidium, Giardia, Cyclospora). En: Guías para la calidad del agua potable. $2^{\mathrm{a}}$ ed. Ginebra, Suiza: OMS. p 70-118.
18. Lieb MS, Zajac AM. 1999. Giardiasis in dogs and cats. Vet Med 94:793-802.

19. Papini R, Cardini $G$ 2006. Evaluation of a rapid Crytosporidium/Giardia inmunochromatgraphic test for diagnosis of giardiasis in dogs. Revue Méd Vét 157: 490-493.

20. Sotelo H, Chávez A, Casas E, Pinedo R, Falcón N. 2013. Giardiasis y criptoporidiasis en caninos de los distritos del cono oeste de Lima Metropolitana. Rev Inv Vet Perú 24: 353-359. doi: 10.15381/rivep.v24i3.2584

21. Sprong H, Caccio SM, Van Der Giessen $\boldsymbol{J W}$. 2009. Identification of zoonotic genotypes of Giardia duodenalis. PLoS Negl Trop Dis 3(12): e558. doi: 10.1371/journal.pntd.0000558.

22. Tello R. 1988. Empleo de una nueva técnica parasitológica rápida de sedimentación espontánea en el diagnóstico de protozoarios y helmintos. En: Simposio Internacional Parasitismo Intestinal en el Hombre. Lima: Sociedad Peruana de Parasitología. p 70.

23. Thompson $\boldsymbol{R}$ C. 2008. Giardiasis: Conceptos modernos sobre su control tratamiento. Ann Nestlé 66: 23-29.

24. Upjohn M, Cobb C, Monger J, Geurden T, Claerebout E, Fox M. 2010. Prevalence, molecular typing and risk factor analysis for Giardia duodenalis infections in dogs in a central London rescue shelter. Vet Parasitol 172: 341-346. doi: 10.1016/j.vetpar.2010.05.010

25. Wang A, Ruch-Gallie R, Scorza V, Lin $P$, Lappin M. 2012. Prevalence of Giardia and Cryptosporidium species in dog park attending dogs compared to non-dog park attending dogs in one region of Colorado. Vet Parasitol 184: 335-340. doi: 10.1016/j.vetpar. 2011.08.019

26. Zárate D, Chávez A, Casas E, Falcón N. 2003. Prevalencia de Giardia sp en caninos (Canis faniliaris) de los distritos del Cono Sur de Lima Metropolitana. Rev Inv Vet Perú 14: 134-139. doi: 10.15381/rivep.v14i2.1617 